\title{
Barium Selective Chemosensing by Diazacrown Ether Naphthalimide Turn-on Fluorophores for Single Ion Barium Tagging
}

\author{
P. Thapa, ${ }^{* \dagger, \ddagger ~ N . ~ K . ~ B y r n e s, ~}{ }^{*, \dagger}$ A. A. Denisenko, ${ }^{\ddagger}$ F.W. Foss, Jr., ${ }^{\ddagger}$ B.J.P. Jones, ${ }^{\dagger}$ J. X. Mao, ${ }^{\ddagger}$ A.D. \\ McDonald, ${ }^{\dagger}$ K. Nam, ${ }^{\ddagger}$ C.A. Newhouse, ${ }^{\ddagger}$ D.R. Nygren, ${ }^{\dagger}$ T.T. Vuong, ${ }^{\ddagger}$ and K. Woodruff ${ }^{\dagger}$ \\ $\dagger$ Department of Physics, University of Texas at Arlington, Arlington, TX 76019 \\ $\ddagger$ Department of Chemistry and Biochemistry, University of Texas at Arlington, Arlington, TX 76019
}

E-mail: pawan.thapa@uta.edu; byrnes.nicholas@mavs.uta.edu

\begin{abstract}
Single molecule fluorescence detection of barium is investigated for enhancing the sensitivity and robustness of a neutrinoless double beta decay $(0 \nu \beta \beta)$ search in ${ }^{136} \mathrm{Xe}$, the discovery of which would alter our understanding of the nature of neutrinos and the early history of the Universe. A key developmental step is the synthesis of bariumselective chemosensors capable of incorporation into ongoing experiments in high-pressure ${ }^{136} \mathrm{Xe}$ gas. Here we report turn-on fluorescent naphthalimide chemosensors containing monoaza- and diaza-crown ethers as agents for single $\mathrm{Ba}^{2+}$ detection. Monoaza-18-crown-6 ether naphthalimide sensors showed sensitivity primarily to $\mathrm{Ba}^{2+}$ and $\mathrm{Hg}^{2+}$, whereas two diaza-18-crown-6 ether naphthalimides revealed a desirable selectivity toward $\mathrm{Ba}^{2+}$. Solution-phase fluorescence and NMR experiments support a photoinduced electron transfer mechanism enabling turn-on fluorescence sensing in the presence of barium ions. Changes in ion-receptor interactions enable effective selectivity between competitive barium, mercury, and potassium ions, with detailed calculations correctly predicting fluorescence responses. With these molecules, dry-phase single $\mathrm{Ba}^{2+}$ ion imaging with turnon fluorescence is realized using oil-free microscopy techniques. This represents a significant advance toward a practical method of single $\mathrm{Ba}^{2+}$ detection within large volumes of ${ }^{136} \mathrm{Xe}$, plausibly enabling a background-free technique to search for the hypothetical process of $0 \nu \beta \beta$.
\end{abstract}

\section{Keywords}

Barium Tagging, Fluorescence Detection, PET Chemosensor, Single Molecule

\section{Introduction}

The development of single barium ion sensing techniques in gaseous or liquid xenon environments is known as "barium tagging" ${ }^{1}$ and is a vibrant area of R\&D in nuclear physics. ${ }^{2,3}$ If realized with high efficiency and at large scales, dry detection of individual $\mathrm{Ba}^{2+}$ in coincidence with well-measured ionization charge deposits in xenon would greatly improve discrimination against relatively copious background processes due to radioactivity, and would enhance discovery reach of searches for the hypothetical radioactive process of $0 \nu \beta \beta$. A robust observation would demonstrate that the neutrino is its own antiparticle, a discovery with major impact for particle and nuclear physics as well as for cosmology. ${ }^{4}$ One possible technical implementation of barium tagging employs single molecule fluorescent imaging (SMFI) with chemosensors that exhibit turn-on fluorescence in response to chelation with barium. ${ }^{5,6}$

Fluorescence-based chemosensors are sensitive analytical tools for rapid chemical and biochemical measurement. ${ }^{7-9}$ Properly incorporated within SMFI experiments, they reveal individual molecular events and fundamentals whose significance is unavailable through bulk analyses. 1,8Naphthalimide fluorophores exhibit many favorable properties for developing new chemosensors. ${ }^{10,11}$ Synthetically tractable 1,8-naphthalimides allow construction of both photoinduced electron transfer (PET) and intramolecular charge transfer (ICT) fluorescent sensors. ${ }^{12}$ Relatively large Stokes shifts and high photostability features in these fluorophores are beneficial for high signal-to-noise ratios. ${ }^{13}$ Crown ethers afford enhanced efficiency in binding to metal ions and can act as switches for PET and ICT based sensors. ${ }^{14}$ As such, 1,8-naphthalimide fluorophores appended with crown ethers have been used as colorimetric and fluorescent sensors for the detection of ions and small molecules that are of great significance in environment, health, and energy. ${ }^{15-18}$ Several 1,8-naphthalimide chemosensors have been developed for detection of cations, anions, and small polar molecules. ${ }^{19-22}$ However, 1,8-naphthalimide based chemosensors with selective response to $\mathrm{Ba}^{2+}$ remain underdeveloped. ${ }^{9,23}$

Selective barium sensing has been demonstrated by a relatively small number of instructive fluorescent chemosensors. ${ }^{9,24-32}$ Barium sensing is not widely studied in clinical settings. Though acute barium exposure can be deadly, diagnoses are rare and often confirmed by inductively-coupled plasma mass spectrometry analysis of patient plasma and urine. ${ }^{33,34}$ Beyond the primary goals of barium tagging in $0 \nu \beta \beta$, sensitive chemosensors for direct detection of $\mathrm{Ba}^{2+}$ would be a promising clinical advance, and some recent reports exist. Nakahara and coworkers pioneered a monoazacryptand receptor with PET switch to pyrene fluorescence that operates in aqueous micellar systems. ${ }^{35,36}$ Crown ether systems invoke termolecular complexes with two crown moieties sandwiched around one $\mathrm{Ba}^{2+} \cdot{ }^{23,37} \mathrm{~A}$ bioinspired G-quadruplex chemosensor provided a rapid detection of $\mathrm{Ba}^{2+} \cdot{ }^{38,39}$ Recently, a fluorescent chemosensor based on phenoxazine system with an ICT turn-on mechanism was reported for $\mathrm{Ba}^{2+}$, where charge transfer was acheived by metal binding specifically to a single amide functional group. ${ }^{40}$ These receptors are instructive, but unsuitable for dry-phase SMFI device design. 
Within the context of R\&D toward a barium-tagging phase of the NEXT program, ${ }^{41-43}$ we previously demonstrated single ion sensitivity to $\mathrm{Ba}^{2+}$ using commercial chemosensors in an aqueous suspension. ${ }^{3}$ Achieving single ion sensitivity mandated the use of a competitive binding agent BAPTA, and used fluorophores incompatible with dry operation. To address the challenge of solventless sensing of barium, a class of dry-phase active fluorescent chemosensors using monoazacrown receptors was demonstrated. ${ }^{44}$ Single-molecule sensitivity of this system described in Ref. ${ }^{44}$ was elusive due to UV excitation of impurities in the substrates and lack of photobleaching transitions to use for single molecule identification. Similar molecules have also recently been shown to be suitable for ratiometric or "bi-color" $\mathrm{Ba}^{2+}$ sensing, ${ }^{45}$ a promising new direction under consideration for barium tagging.

In this paper we report a study on the synthesis, characterization, analysis, and computational modeling of a new family of monoazacrown and diazacrown naphthalimide fluorescent sensors (Fig. 1) with enhanced selectivity and sensitivity to $\mathrm{Ba}^{2+}$. These visible fluorescent probes are amenable to SMFI microscopy in dry environments and consequently allowed us to resolve individual $\mathrm{Ba}^{2+}$ ions without a competitive binding agent. This extends the functionality of the dyes described in Ref., ${ }^{44}$ with visible excitation proving to be a critical ingredient in overcoming fluorescent backgrounds of glass and quartz substrates at the level required for single molecule sensitivity. We also report on computational models that offer reliable predictability of target ion- and binding-site dependent fluorescent response within this family, a capability that will accelerate explorations of structure-function relationships in dry-phase SMFI probes.

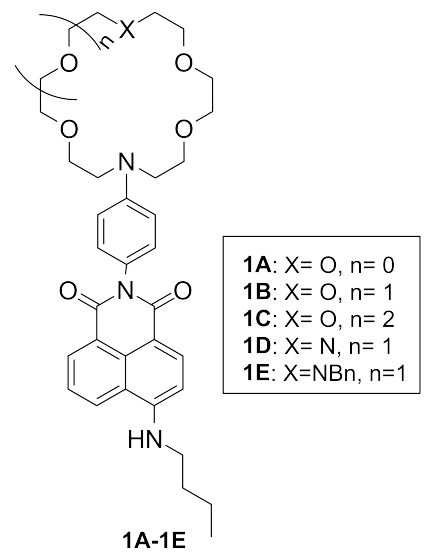

Figure 1: Napthalimide derivatives used in this study

\section{Experimental Section}

\section{$2.1 \quad$ Synthesis}

The synthesis of naphthalimide derivatives $\mathbf{1 A - 1 E}$ is shown in Scheme 1. Monoazacrown ethers 2A-2C were assembled as previously described. ${ }^{44,46}$ Diaza-18-crown-6 ethers were prepared by the modification of methods from a previous report. ${ }^{47}$ Complete details and chemical characterization can be found in the supplementary information.

\subsection{Sample preparation for solution-phase studies}

Stock solutions of probe molecules were prepared at $1 \times 10^{-3} \mathrm{M}$ concentration in acetonitrile solvent. Stock solutions of metal perchlorates were made at $1 \times 10^{-2} \mathrm{M}$ concentration in 9:1 acetonitrile/water mixture. UV-Vis studies were performed with and without $\mathrm{Ba}^{2+}$ in acetonitrile, dichloromethane, acetone, and ethanol solvent with probe 1B $\left(10 \times 10^{-6} \mathrm{M}\right)$ and barium perchlorate $\left(50 \times 10^{-6} \mathrm{M}\right)$. Fluorescence titration and metal ion selectivity studies were performed by maintaining a final concentration of probe molecule at $1 \times 10^{-6} \mathrm{M}$ concentration. Metal ion selectivity studies were conducted by maintaining metal perchlorate concentration at $5 \times 10^{-6} \mathrm{M}$. Competitive fluorescence experiments were performed using $2 \times 10^{-6} \mathrm{M}$ of the probe 1D with $5 \times 10^{-6} \mathrm{M}$ metal perchlorate solution in acetonitrile solvent. Job's plot experiments were performed by maintaining a total concentration of the probe and barium perchlorate solution at $20 \times 10^{-6} \mathrm{M}$. Samples were incubated for two minutes in the dark before measuring fluorescence intensity. ${ }^{1} \mathrm{H}$ NMR titration experiments were conducted with the concentration of probe at $1 \times 10^{-2} \mathrm{M}$ and metal perchlorate (barium, potassium, and mercury) at $1 \times 10^{-2} \mathrm{M}$ and $5 \times 10^{-2} \mathrm{M}$ in acetonitrile- $d_{3}$ solvent at room temperature.

\subsection{Slide preparation for dry-phase fluores- cence}

Dry samples of probe 1D on glass slides for dry-phase fluorescence studies were prepared as follows: An acetonitrile solution of barium selective probe $\mathbf{1 D}$ was mixed into cyanoacrylate glue to desired concentration $\left(10 \times 10^{-12} \mathrm{M}\right.$ to $\left.10 \times 10^{-6} \mathrm{M}\right) .30 \mu \mathrm{L}$ of this solution was applied to a glass microscope slide and then dried in an oven for five minutes at $373 \mathrm{~K}$ to set into a thick solid layer. Fluorescence response from three slides was measured with excitation at $430 \mathrm{~nm}$, before and after washing with a $50 \mu \mathrm{L}$ of $1 \times 10^{-3} \mathrm{M}$ barium perchlorate in acetonitrile and then drying. Each slide was then re-scanned three times, and the fluorescence response was averaged to yield a mean response.

\subsection{Microscopy details}

The probe $1 \mathrm{D}$ was suspended at a concentration of $10^{-11} \mathrm{M}$ within the cyanoacrylate matrix and analyzed via fluorescence microscopy. Excitation is delivered by a Supercontinuum laser with output selected by acousto-optical tunable filter. For the studies performed here, several output lines were superposed between $420 \mathrm{~nm}$ and $437.5 \mathrm{~nm}$, to maximize output power and cover the excitation band of 1D. Laser light was cleaned by passing through a beam-expander and cutting off the outer, non-uniform edge with an iris, generating a circular and approximately Gaussian beam profile. The beam was then redirected through a $500 \mathrm{~nm}$ shortpass filter to reduce incident background light, reflected off a $505 \mathrm{~nm}$ dichroic mirror, and focused through a $100 \times$ aircoupled microscope objective $(\mathrm{NA}=0.95)$ for a final power output of $125 \mathrm{~mW}$ to excite the $1 \mathrm{D}$ matrix in epifluorescent mode. The objective collected the resultant Stoke's shifted fluorescent emissions, which were then passed back through the dichroic mirror and a $500 \mathrm{~nm}$ long-pass filter, again for background reduction, and collected via a Hamamatsu EMCCD camera, which acquired one image every half-second into 300-image sequences. 
Microscope images were processed using an algorithm that first filters the image in Fourier space to remove slowly sloping backgrounds and then sums the sequence over the full imaging time. After filtering, single molecule candidates can be identified after subtracting the background profile, which is found by blurring the summed image via a Gaussian filter. Single molecule candidates are identified as points with intensities in excess of $3 \sigma$ above the background. Once the candidates are identified, their locations on the raw images are analyzed as a function of time for the duration of the sequence to produce fluorescent trajectories. These trajectories are scanned for an instantaneous drop or "single step" profile, which is the hallmark characteristic of a single molecule undergoing a discrete photo-bleaching process.

\subsection{Computation details}

Molecular structures were optimized using M06- $2 \mathrm{X}^{48}$ functional with the SDD ${ }^{4-52}$ effective core potential basis set for heavy metal atoms (barium, mercury) and def2-SVP ${ }^{53,54}$ basis set for other atoms, followed by frequency analysis to confirm the nature of their energy minima (no imaginary frequency). Calculations with time-dependent densityfunctional theory (TDDFT) ${ }^{55}$ were carried out to reveal the orbitals involved in the observed fluorescence events. SMD ${ }^{56}$ or $\mathrm{PCM}^{57}$ solvation models were used to incorporate solvent effects with acetonitrile as the solvent. All calculations were performed using the Gaussian 09 program. ${ }^{58}$

\section{Results and discussions:}

\subsection{Synthesis}

Scheme 1 represents the synthesis of probes 1A-1E. In brief, nucleophilic aromatic substitution between 4fluoronitrobenzene and mono/diaza crown ethers, prepared by modification of our previous work, ${ }^{44}$ gave the desired 4-nitroaniline derivatives 3A-3D. Reducing 4fluoronitrobenzene to one equivalent was critical in the case of diaza-18-crown-6 ether 2D. Nitro reduction by $\mathrm{Pd}-$ catalyzed hydrogenolysis, followed by condensation with commercially available 4-bromonaphthalic anhydride resulted in the desired bromonaphthalimide products 4A-4D. Initial attempts for the final nucleophilic aromatic substitution reaction of bromonaphthalimide with $n$-butylamine in 2-methoxyethanol solvent led to poor yields of the desired aminonaphthalimides. However, $N$-methyl-2-pyrrolidone (NMP) acting as a basic solvent enabled the bromide substitution to afford all desired aminonaphthalimides 1A-1D. Compound $\mathbf{1 E}$ was prepared by nucleophilic substitution by dropwise addition of benzyl bromide into a solution containing $1 \mathrm{D}$ and potassium carbonate. Overall, this synthetic approach led to the efficient preparation of the desired naphthalimide fluorescent probes 1A-1E.

\subsection{UV-Vis studies}

UV-Vis spectra of all synthesized 1,8-naphthalimide based fluorescent probes (1A-1E) were recorded in acetonitrile solvent at room temperature. Major absorbances were centered between 200-300 $\mathrm{nm}$ and 380-480 $\mathrm{nm}$ - consistent with 4amino-1,8-naphthalimide chromophores. ${ }^{11}$ Solutions doped with $\mathrm{Ba}^{2+}$ provided no or negligible shifts on UV-Vis absorbances in all probes (SI Fig. S2). These effects were also encountered in dichloromethane, acetone, and ethanol

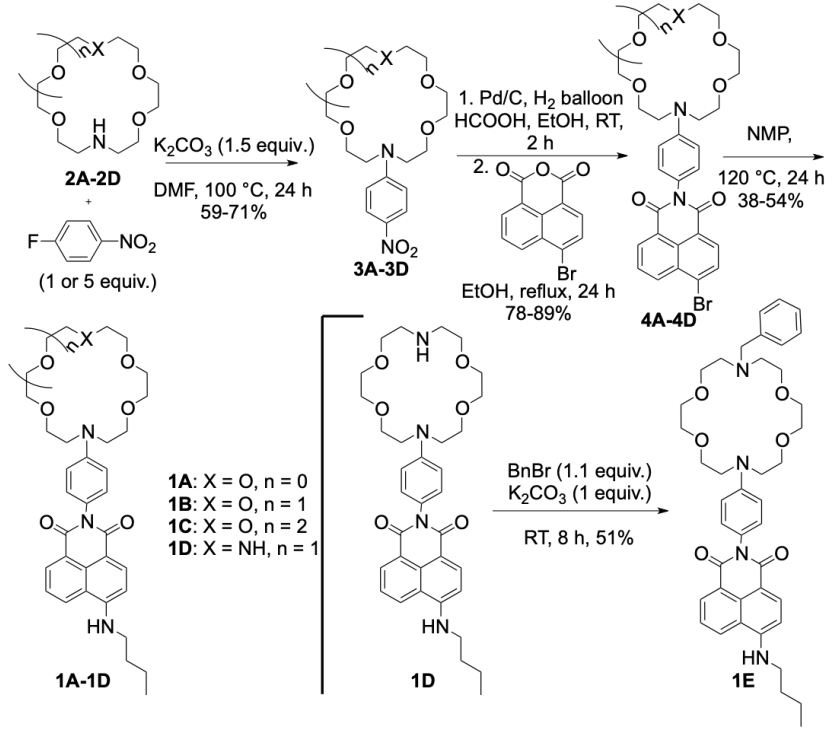

Scheme 1: Synthesis of 4-amino-1,8-naphthalimide sensors.

(SI Fig. S3). $\mathrm{Ba}\left(\mathrm{ClO}_{4}\right)_{2}$ addition led to a concentration dependent increase in fluorescence centered near $530 \mathrm{~nm}$ in probes 1B, 1D, and 1E, when excited at $\lambda_{\max }$ obtained from UV-Vis spectrum, suggesting turn-on fluorescence by changes to photoinduced electron transfer (PET). ${ }^{17}$ Similarly, fluorescence analysis as a function of absorption wavelength from 430-460 $\mathrm{nm}$ showed no significant change in the emission intensity profile (SI Fig. S4).

\subsection{Fluorescence response and metal ion selectivity}

Fluorescent response to cationic analytes was determined for each of the synthesized probes $(1.0 \mu \mathrm{M}$ in acetonitrile). Stock solutions of perchlorate salts were prepared in 9:1 mixture of $\mathrm{MeCN} / \mathrm{H}_{2} \mathrm{O}$ to achieve complete dissolution. The final concentration of perchlorate solution was maintained at $5 \mu \mathrm{M}$ to achieve 1:5 concentration ratio of probe to ion analyte. The selectivity studies are summarized in Fig. 2, top panel with emission at $530 \mathrm{~nm}$. Molecular probes $\mathbf{1 A}$ and $\mathbf{1 C}$ are not effective optical sensors for almost all tested ions. A small two-fold response was found in the case of calcium with probe $\mathbf{1 A}$. In contrast, 1B showed excellent sensitivity towards $\mathrm{Ba}^{2+}$ and $\mathrm{Hg}^{2+}$ in addition to a lesser response to $\mathrm{Ca}^{2+}$. In the presence of $\mathrm{Ba}^{2+}$ and $\mathrm{Hg}^{2+}$ solutions, ca. 30-fold increases in fluorescence intensities were observed. $\mathrm{Ca}^{2+}$ and $\mathrm{Cd}^{2+}$ ions also showed eight-fold and five-fold increase, respectively for probe $\mathbf{1 B}$.

Two novel 1,8-naphthalimide derivatives containing diazacrown ether binding domains $1 \mathbf{D}$ and $\mathbf{1 E}$ were found to have excellent selectivity to $\mathrm{Ba}^{2+}$. $\mathbf{1 D}$ and $\mathbf{1 E}$ showed solution phase emission enhancements of 11 -fold and 22-fold, respectively, in the presence of $\mathrm{Ba}^{2+}$. Among different metal ions $\left(\mathrm{Mg}^{2+}, \mathrm{Ca}^{2+}, \mathrm{Cu}^{2+}, \mathrm{Zn}^{2+}, \mathrm{Cd}^{2+}, \mathrm{Hg}^{2+}, \mathrm{Ba}^{2+}, \mathrm{Li}^{+}, \mathrm{Na}^{+}\right.$, $\mathrm{K}^{+}, \mathrm{Rb}^{+}, \mathrm{Ag}^{+}, \mathrm{Cs}^{+}$, and $\left.\left(\mathrm{CH}_{3}\right)_{4} \mathrm{~N}^{+}\right)$studied, only $\mathrm{Ba}^{2+}$ showed significant increased fluorescence for probe 1D and 1E. Other metal ions showed little to none $(<2 \times)$ fluorescence intensity. Normalized fluorescence responses highlight selectivity by ignoring differences in absolute brightness, and show $\mathbf{1 D}$ and $\mathbf{1 E}$ with highest selectivity For $\mathrm{Ba}^{2+}$ (Fig. 2, bottom panel). Barium sensitivity remained in competitive fluorescence experiments (Fig. 3). The barium selectivity of 


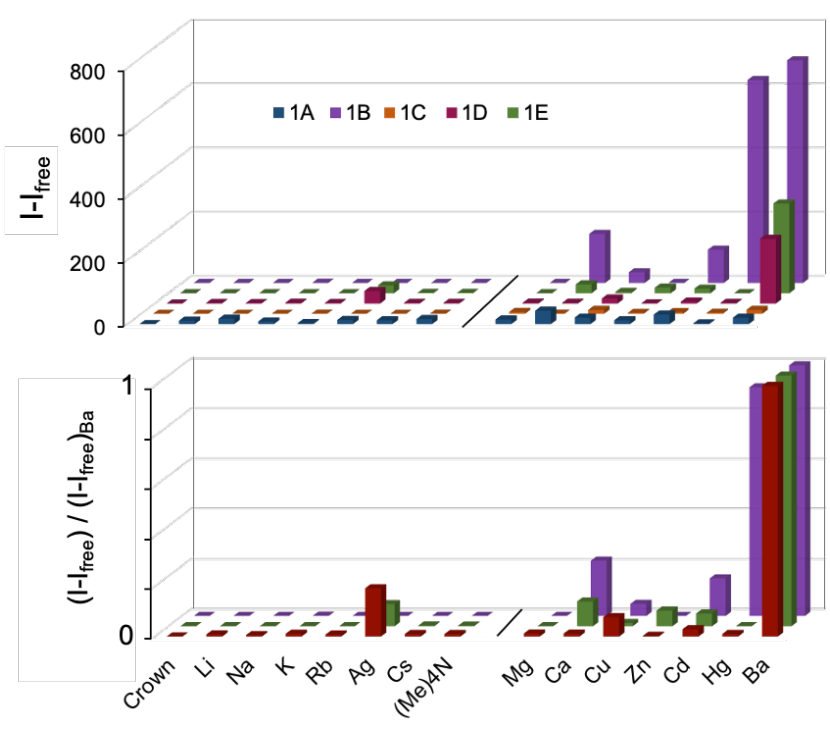

Figure 2: Top: ion perchlorate $\left(5 \times 10^{-6} \mathrm{M}\right)$ response to $\mathbf{1 A}$ $\mathbf{1 E}\left(1 \times 10^{-6} \mathrm{M}\right)$ in MeCN solution. Bottom: fluorescence response of $\mathbf{1 B}, \mathbf{1 D}$, and $\mathbf{1 E}$ with different cation solutions normalized to fluorescence intensity for barium perchlorate.

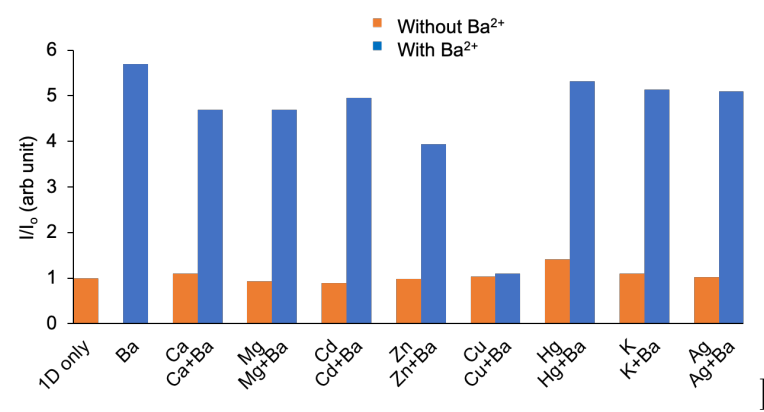

Figure 3: Fluorescence response of $\mathbf{1 D}\left(2 \times 10^{-6} \mathrm{M}\right)$ to $\mathrm{Ba}^{2+}\left(5 \times 10^{-6} \mathrm{M}\right)$ in the presence of other metal ions (Ions, $\left.5 \times 10^{-6} \mathrm{M}\right)$ in acetonitrile. Slit width $2.5 / 5.0$.

probe 1D is observed in all cases, except with copper, where direct probe oxidation is likely. Combined, these results are clear evidence of increased selectivity of two novel optical sensors $\mathbf{1 D}$ and $\mathbf{1 E}$ to $\mathrm{Ba}^{2+}$.

\subsection{Fluorescence titration and binding studies}

Fluorescence titration curves presented in Fig. 4A, SI Fig. S5A, and SI Fig. S6A show that the emission maxima are obtained before five equivalents of $\mathrm{Ba}^{2+}$ are added for probes $\mathbf{1 E}, \mathbf{1 B}$, and $\mathbf{1 D}$ respectively. Titration studies performed with probes $\mathbf{1 B}, \mathbf{1 D}$, and $\mathbf{1 E}$ with an increasing concentration of barium perchlorate in acetonitrile gave dissociation constant values $\left(\mathrm{k}_{d}\right)$ of $0.72 \times 10^{-6} \mathrm{M}, 0.59 \times 10^{-6} \mathrm{M}$ and $0.75 \times 10^{-6} \mathrm{M}$, respectively. These low dissociation constants reveal effective binding between each probe and $\mathrm{Ba}^{2+}$. Solution phase limits of detection (LOD) were calculated using linear fitting curves of fluorescence titration data and the formula $\mathrm{LOD}=3 \sigma / \mathrm{k}$, where $\sigma$ is the standard deviation of the blank sample without barium and $\mathrm{k}$ is the slope between intensity versus barium concentration, ${ }^{59}$ resulting in $0.0288 \times 10^{-6} \mathrm{M}, 0.074 \times 10^{-6} \mathrm{M}$, and $0.006 \times 10^{-6} \mathrm{M}$ detection limits for probe $\mathbf{1 E}, \mathbf{1 B}$, and $\mathbf{1 D}$, respectively. Fig. 4B,
SI Fig. S5B, and SI Fig. S6B show good linear correlation for probes $\mathbf{1 E}\left(\mathrm{R}^{2}=99.5\right), \mathbf{1 B}\left(\mathrm{R}^{2}=98.5\right)$, and $\mathbf{1 D}\left(\mathrm{R}^{2}\right.$ $=99.1)$, respectively. Overall results show that the probe 1B, 1D and 1E can detect $\mathrm{Ba}^{2+}$ in solution at nanomolar concentrations and therefore, can be highly practical fluorescent sensors for selective detection of $\mathrm{Ba}^{2+}$. The stoichiometry of binding for molecular probes $\mathbf{1 B}$ and $\mathbf{1 E}$ with barium were calculated by Job's method. The results, presented in supplementary SI Fig. S5C and Fig. 4C, show the inflection point of two linear fitting curves at 0.46 and 0.50 mole fraction for complex $\mathbf{1 B}-\mathbf{B a}^{2+}$ and $\mathbf{1 E -} \mathbf{B a}^{2+}$ respectively. This indicates a 1:1 binding stoichiometry between probes $\mathbf{1 B}$ and $\mathbf{1 E}$ with $\mathrm{Ba}^{2+}$. Additionally, complex 1B$\mathbf{B a}^{2+}$ was observable in high resolution mass spectrometric (HRMS) analysis of solution of $\mathbf{1 B}$ and barium perchlorate (SI Fig. S5D).

\section{$3.5{ }^{1} \mathrm{H}$ NMR studies}

Complexation between $\mathrm{Ba}^{2+}$ and fluorescent probes 1B, 1D, and $\mathbf{1 E}$ were further demonstrated by ${ }^{1} \mathrm{H}$ NMR experiments performed with 1:1 and 1:5 molar ratio of probe and $\mathrm{Ba}\left(\mathrm{ClO}_{4}\right)_{2}$ in acetonitrile- $d_{3} . \mathrm{Ba}^{2+}$ addition resulted in downfield shifts of $N$-phenyl-aza crown ether protons, while the chemical shift values of protons in naphthalimide fluorophore group have minimal or no change (Fig. 4D). Noticeably, an upfield shift in $\alpha$-protons of the anilino nitrogen in azacrown ether was also observed (H15 and H26 in Fig. 4D) in all molecular probes (for $\mathbf{1 B}$ and $\mathbf{1 D}$, see SI Fig. S7), suggesting that the nitrogen atom of $N$-phenylaza crown ether is not directly involved in ion binding. Similarly, the effect of $\mathrm{Ba}^{2+}$ on N-benzyl group in probe $\mathbf{1 E}$ was observed, as seen with downfield shifts of both aromatic and benzylic protons (Fig. 4D). Additionally, in fluorescent probe $1 \mathbf{D}$ and $1 \mathbf{E}$, all $\alpha$-protons of two nitrogen atoms in the diazacrown ether structure showed upfield shift indicating no interaction between both nitrogen atom of diazacrown and $\mathrm{Ba}^{2+}$. The results obtained from ${ }^{1} \mathrm{H}$ NMR experiments with probes clearly show the binding of $\mathrm{Ba}^{2+}$ on the receptor crown ether units with no significant binding on naphthalimide fluorophore units. Comparative ${ }^{1} \mathrm{H}$ NMR studies were carried out with $\mathrm{Ba}^{2+}, \mathrm{Hg}^{2+}$ and $\mathrm{K}^{+}$in the case of probe $\mathbf{1 B}$, which show distinct features in the NMR spectra. With $\mathrm{Hg}^{2+}$ addition, all monoazacrown methylene protons were deshielded, indicating binding of nitrogen atom of N-phenyl-aza crown ether with $\mathrm{Hg}^{2+}$ (SI Fig. S8). With $\mathrm{K}^{+}$, however, no observable change was found in the chemical shift values of the protons of both naphthalimide and azacrown ether moieties (SI Fig. S9).

\subsection{Computational studies}

Computational models were studied using TDDFT to predict the fluorescence behaviour of sensors in response to ions in acetonitrile solution. As a test suite we considered molecules $\mathbf{1 B}$ and $\mathbf{1 E}$, both unchelated and chelated with $\mathrm{Ba}^{2+}, \mathrm{K}^{+}$, and $\mathrm{Hg}^{2+}$. The results support the proposed PET-mediated off-state within unchelated mono- and diazacrown ether naphthalimides, which is turned-on by chelation-enhanced fluorescence (CHEF) upon ion binding. In the unbound 1B species (Fig. 5A), excitation of the naphthalimide fluorophore is governed by a HOMO-1 to LUMO transition based on orbital analysis. The electrons in the interstitial $\mathrm{HOMO}_{\text {free }}$ orbital of the receptor (highlighted) are localized around the $N$-aryl unit and energetically well-positioned to quench the excited state before fluo- 

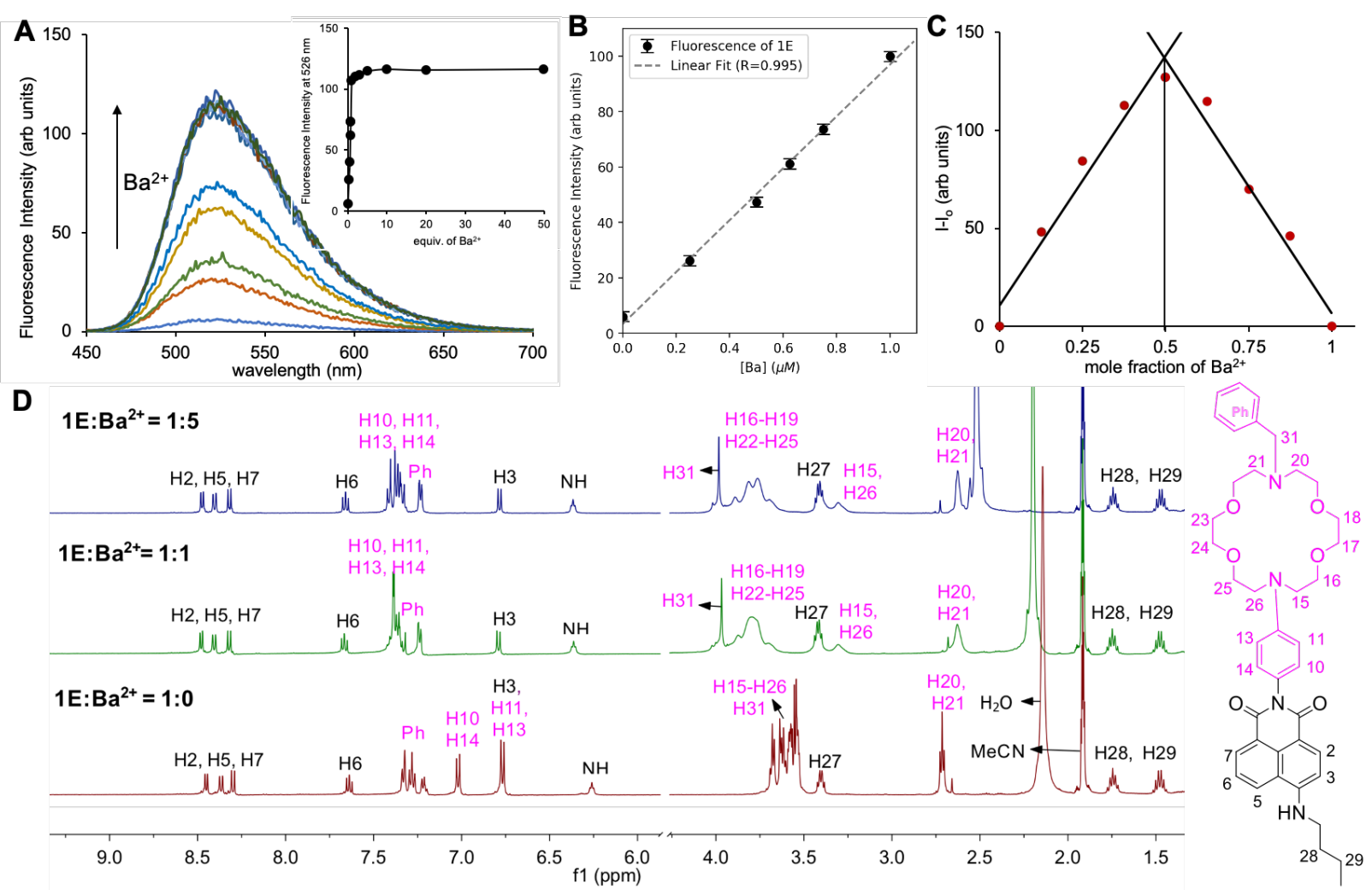

Figure 4: A: Fluorescence titration spectra of $\mathbf{1 E}\left(1 \times 10^{-6} \mathrm{M}\right)$ upon addition of $\mathbf{B a}^{2+}\left(0-50 \times 10^{-6} \mathrm{M}\right)$ in acetonitrile $\left(\lambda_{e x}=\right.$ $430 \mathrm{~nm})$. B: Linearity of fluorescence intensity at $526 \mathrm{~nm}$ for $\mathbf{1 E}\left(1 \times 10^{-6} \mathrm{M}\right)$ in acetonitrile as a function of the concentration

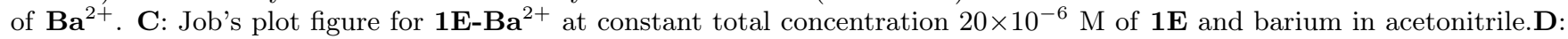
${ }^{1} \mathrm{H}$ NMR titration spectra of probe $\mathbf{1 E}$ with the addition of 0.0 (red trace), 1.0 (green trace), and 5.0 (blue trace) equiv. of $\mathrm{Ba}^{2+}$ in acetonitrile- $\mathrm{d}_{3}$. Inset shows the plot of fluorescence change as a function of $\mathbf{B a}^{2+}$ concentration. Slit width $2.5 / 5.0$

rescence transition can occur from LUMO to $\operatorname{HOMO}(-1)$, by a traditional PET mechanism. Upon barium chelation, however, the $\mathrm{HOMO}_{\text {free }}$ level is drastically stabilized by mixing with $\mathrm{Ba}^{2+}$ and becomes the new HOMO-1 (Fig 5B), allowing the fluorophore-centered HOMO and LUMO levels of the barium bound species to participate in fluorescence without PET-quenching.

As $1 \mathrm{~B}$ responds to $\mathrm{Hg}^{2+}$, but not to $\mathrm{K}^{+}$, we examined the arrangement of frontier molecular orbitals when complexed to these cations. Chelation is effective and lowers the critical PET-enabling $\mathrm{HOMO}_{\text {free }}$ orbital through binding to both ions. However, in the case of $\mathrm{K}^{+}$, PET quenching is still possible (Fig 5C), predicting a non-fluorescence response to $\mathrm{K}^{+}$consistent with experiment. $\mathrm{Hg}^{2+}$ chelation results in notable lowering of the HOMO, LUMO, and LUMO +1 energy states relative to the free chemosensor(Fig $5 \mathrm{D})$; the LUMO and LUMO +1 states show substantial lowering in energy including a reordering of states, not unusual among heavy atom binding. ${ }^{60}$ Excitation of the fluorophorecentered electrons (HOMO to LUMO +1 ) are not quenched by PET. Thus CHEF occurs with $\mathrm{Hg}^{2+}$ addition to $\mathbf{1 B}$, similar to the barium chelation, albeit with a different rearrangement of orbital energies.

Analysis of the selective diazacrown ether chemosensor $\mathbf{1 E}$ showed similarity to $\mathbf{1 B}$ when free and bound to $\mathrm{Ba}^{2+}$ and $\mathrm{K}^{+}$, especially with respect to the critical relative location of the $\mathrm{HOMO}_{\text {free }}$ orbital (highlighted in Fig. 6). However, $\mathrm{Hg}^{2+}$ binding resulted in a weaker effect on the $\mathrm{HOMO}_{\text {free }}$ orbital, and the fluorescence of $\mathrm{Hg}^{2+}$ bound species is still quenched by internal PET from HOMO to HOMO-1. Over- all, the theoretical studies support experimental results for the enhanced selectivity of $\mathbf{1 E}$ to the $\mathrm{Ba}^{2+}$.

In conclusion, the theoretical models correctly predict the observed switch-on fluorescence patterns across all ions and species tested. These are summarized in Table 1.

Table 1: Summary of computational results compared with experimental observations of switch-on fluorescence.

\begin{tabular}{|c|c|cc|}
\hline Probe & Ion & $\begin{array}{c}\text { Fluorescence } \\
\text { predicted }\end{array}$ & $\begin{array}{c}\text { Fluorescence } \\
\text { observed }\end{array}$ \\
\hline \hline $\mathbf{1 B}$ & - & No & No \\
$\mathbf{1 B}$ & $\mathrm{Ba}^{2+}$ & Yes & Yes \\
$\mathbf{1 B}$ & $\mathrm{K}^{+}$ & No & No \\
$\mathbf{1 B}$ & $\mathrm{Hg}^{2+}$ & Yes & Yes \\
\hline $\mathbf{1 E}$ & - & No & No \\
$\mathbf{1 E}$ & $\mathrm{Ba}^{2+}$ & Yes & Yes \\
$\mathbf{1 E}$ & $\mathrm{K}^{+}$ & No & No \\
$\mathbf{1 E}$ & $\mathrm{Hg}^{2+}$ & No & No \\
\hline
\end{tabular}

\subsection{Dry SMFI of $\mathrm{Ba}^{2+}$}

To demonstrate single ion sensing in a dry environment, compound 1D was suspended in cyanoacrylate polymer. Polyvinylacetate and polystyrene matrices were initially investigated with relatively poor solvochromatic effects on bulk fluorescence. Barium-induced fluorescence was first verified by spectrophotometry in bulk matrix. A strong fluorescent response was observed across all three tested locations on three slides, though with significant variability 


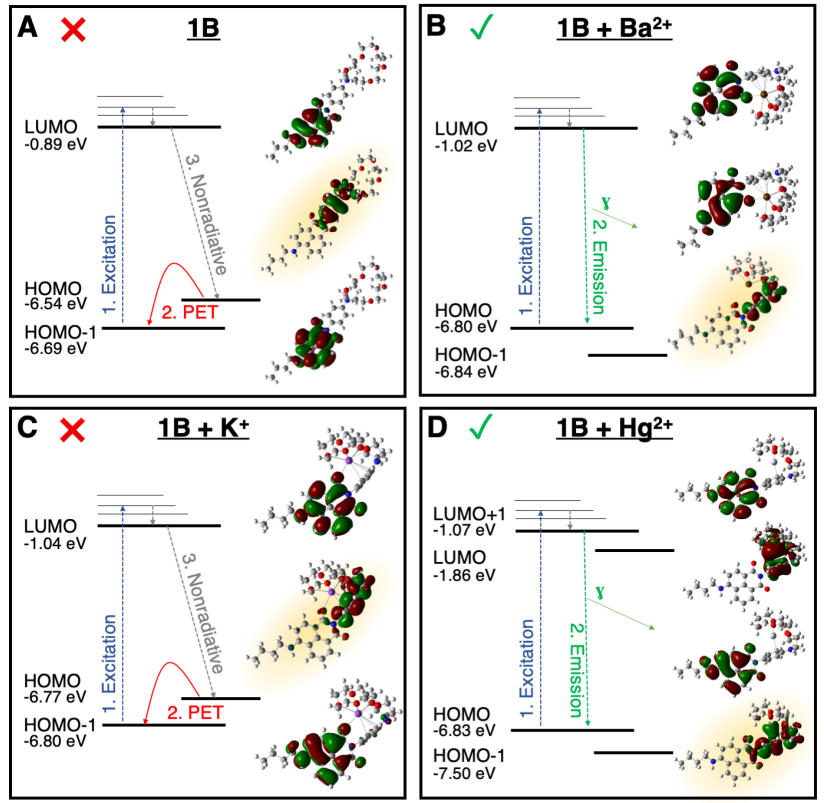

Figure 5: Simulated orbitals of 1B. The mechanism of PET and $\mathrm{CHEF}$ is illustrated by the change in energy of the nitrogen-dominated orbital, highlighted, upon binding various cations.

observed due to imperfect uniformity of layer deposition. Fig. 7A shows the background-subtracted response of slides with no added $\mathrm{Ba}^{2+}$, and after $\mathrm{Ba}^{2+}$ wash. Fluorescence is normalized to activity on the rising edge of the excitation peak before background subtraction. Mean and standard deviation are shown as dashed and filled regions, in addition to individual response curves. Also shown overlaid is a control measurement using a pure solvent wash-resulting in slightly reduced fluorescence, likely due to removal of some ionic contamination or fluorescent emitters from the surface. These data demonstrate that fluorescent response to $\mathrm{Ba}^{2+}$ is maintained in the dry phase, as observed in our past work with molecules of this family. ${ }^{44}$

The imaging modality for SMFI in this paper is aircoupled epifluorescent microscopy. This is distinct from prior work ${ }^{3}$ on SMFI imaging of $\mathrm{Ba}^{2+}$ in solution, which used Total Internal Reflection Fluorescence (TIRF). TIRF offers combined benefits of reduced background through by exciting only at the glass-sample interface; and oil couplings that allow for high numerical aperture imaging. The oil required for through-objective TIRF, however, is likely problematic within the high purity xenon environment of our target application. While the oil-free imaging modality used here introduces additional practical challenges, realization of single $\mathrm{Ba}^{2+}$ imaging in this way encapsulates an important practical step toward application in time projection chambers.

A set of slides was prepared for single-ion level imaging of barium, with compound 1D suspended at $10 \mathrm{pM}$ concentration in the cyanoacrylate matrix. Slides were washed with a $1 \mathrm{nM} \mathrm{Ba}^{2+}$ solution. A stark increase in the net fluorescence intensity of the sample was observed upon addition of $\mathrm{Ba}^{2+}$. Three slides were tested, with image sequences taken across three locations both before and after the barium solution was introduced. The increase in intensity was quantified via raw pixel histogram for each slide, shown in Fig. 7 B. A robust increase in bright pixels was observed in all cases,

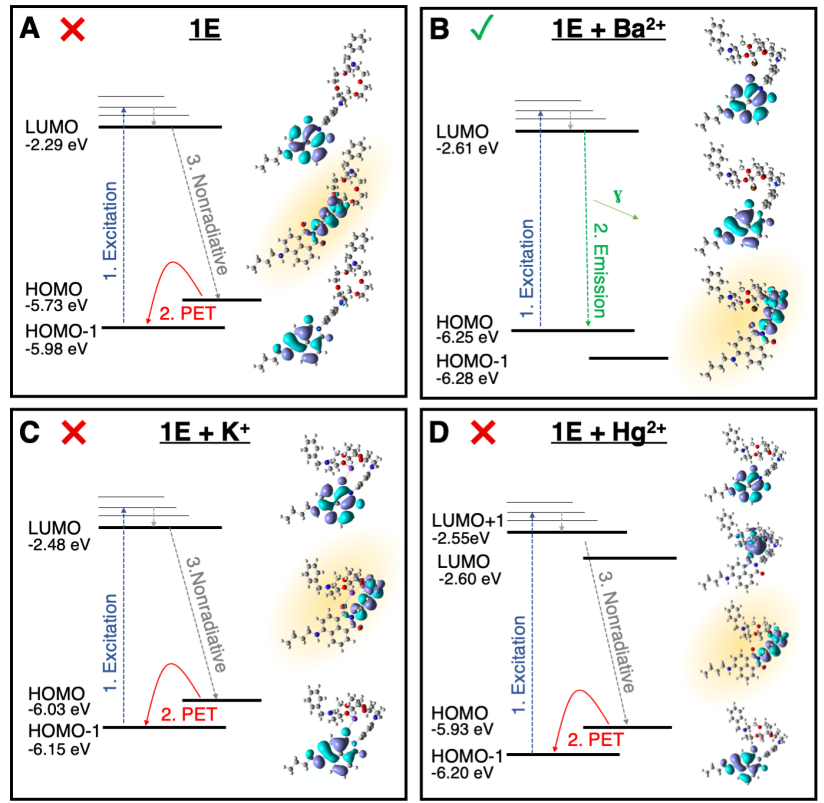

Figure 6: Simulated orbitals of 1E. Enhanced $\mathrm{Ba}^{2}+$ selectivity is illustrated by comparing changes in energy of the nitrogen-dominated orbital, highlighted.

indicative of turn-on fluorescence.

The detected fluorescence in these images originates from molecules at various distances from the focal plane, with near-focus points appearing very bright, and out-of-plane candidates dimmer. Both before and after addition of $\mathrm{Ba}^{2+}$, an array of spots of various brightness can be observed, undergoing discrete photo-bleaching transitions over time, with the number dramatically enhanced in the barium-added images. An example of a before/after comparison is shown in Fig. 7 C.i, where in both cases, bright points corresponding to distinct barium-1D complexes can be visually identified.

Using a sample with $10 \mathrm{pM}$ barium perchlorate solution applied to the $10 \mathrm{pM}$ sensor matrix, the interpretation of these bright emitters as single molecules was confirmed. Fig. 7 C.ii shows an example image processed and filtered according to the algorithm described in the methods section, which is used as input to time-series analysis. The well localized candidate spots in this image, an example of which is shown isolated in Fig. $\mathbf{7} \mathbf{D}$, are observed to exhibit discrete photo-bleaching transitions, as shown in Fig. 7 E. This behaviour, also observed visually for the bright spots in the denser samples, as well in previous work in solution phase ${ }^{3}$ confirms the single molecule interpretation of these fluorescent emitters.

\subsection{Toward $\mathrm{Ba}^{2+}$ tagging in Xenon}

An aspect that remains undemonstrated here is the capture of $\mathrm{Ba}^{2+}$ within an environment of high pressure xenon gas. Our past theoretical work ${ }^{61}$ demonstrated that solvationlike effects with xenon are expected, with the shell configuration depending on gas temperature and pressure. The xenon shell typically contains 7-10 xenon atoms for $\mathrm{Ba}^{2+}$ at 10-15 bar. Capture by a molecular layer must free $\mathrm{Ba}^{2+}$ from this shell of accompanying spectator atoms, typically held at a binding energy of $3-4 \mathrm{eV}$. The $\mathrm{Ba}^{2+}$ binding energy of the molecules developed in this work is calculated to be around $0.2 \mathrm{eV}$ in MeCN. However, calculations of the 

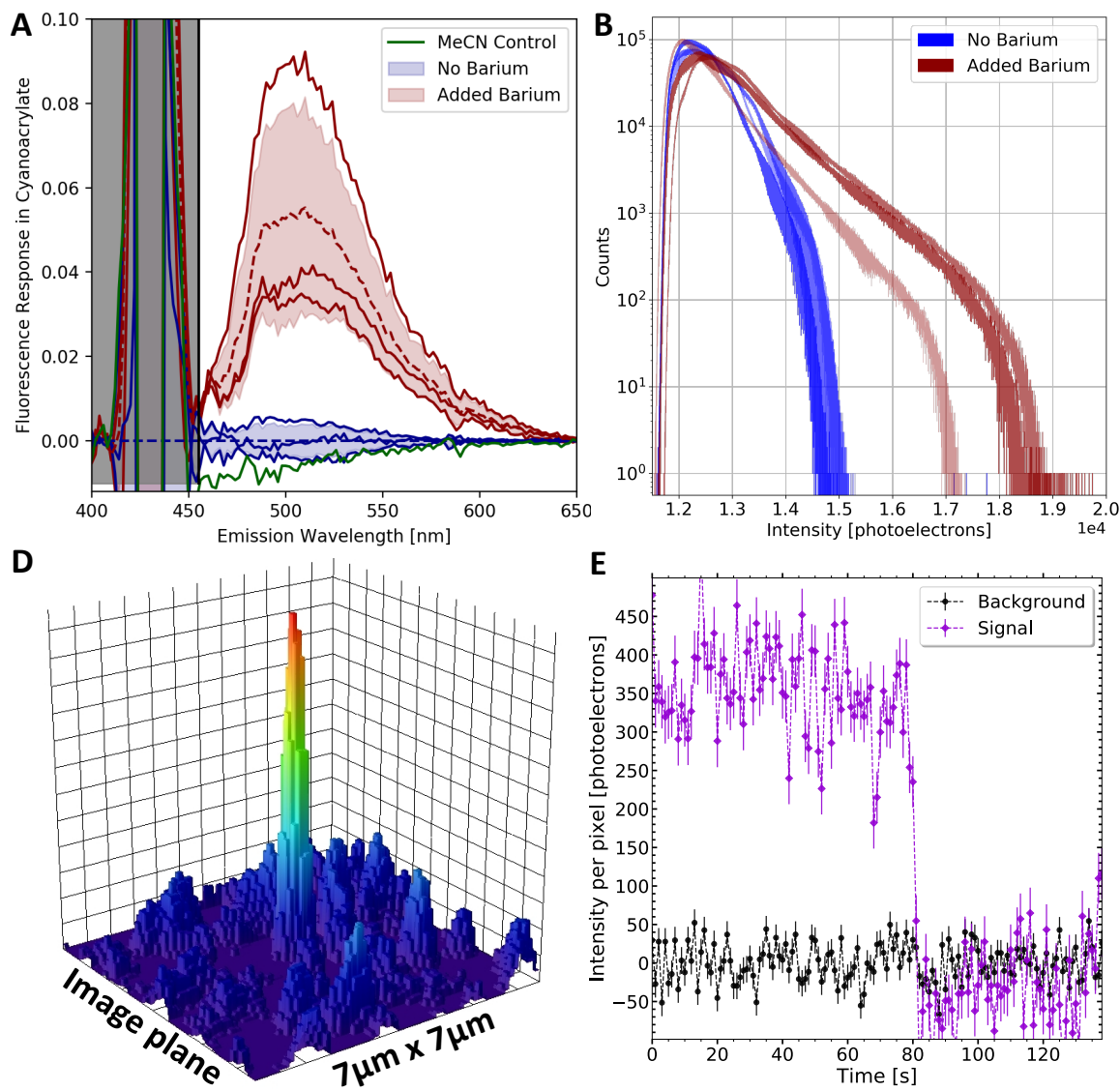

$\mathbf{E}$

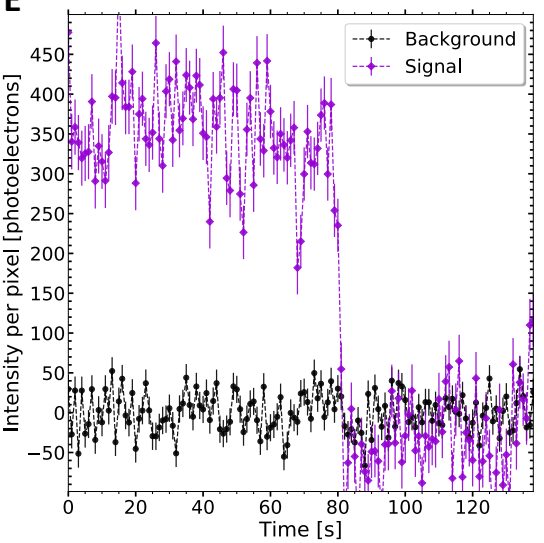

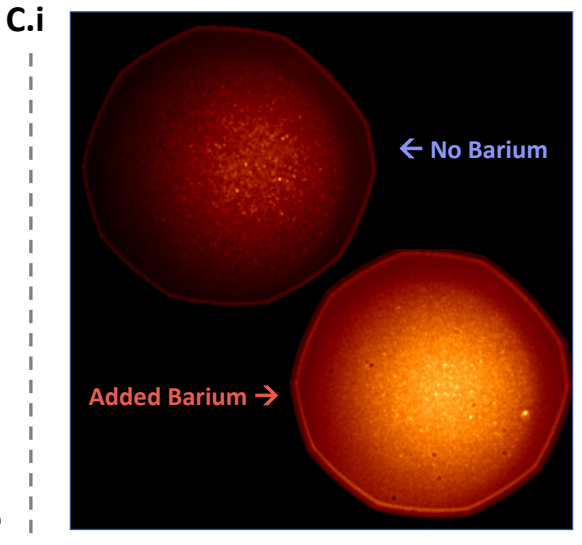

C.ii

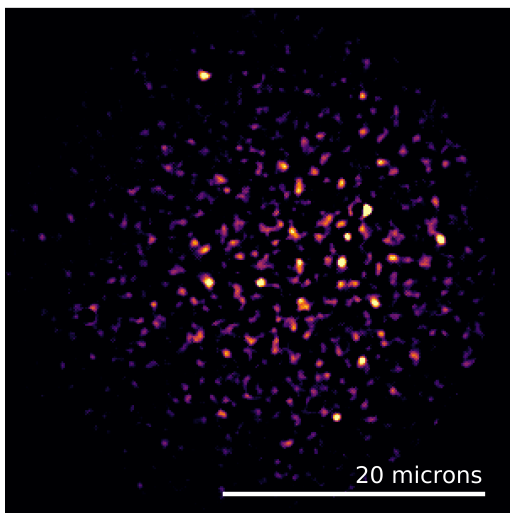

Figure 7: Solid-matrix measurements of barium sensing fluorophore 1D. A: bulk spectrophotometry in dried cyanoacrylate matrix; B: Raw pixel histogram of three 1D coated slides imaged at the single molecule level; C: Single molecule level microscope images of the 1D layer with and without $\mathrm{Ba}^{2+}$ (i) and with $\mathrm{Ba}^{2+}$ after background removal (ii); D: A specific single reconstructed barium-chelated 1D molecule; E: Barium-chelated 1D molecule fluorescence time trajectory showing single-step photo-bleaching characteristic of SMFI detection.

binding affinity in the gas phase, removing polarization effects of the solvent, show a much enhanced binding energy of $-10.5 \mathrm{eV}$. This is sufficient to extract the target $\mathrm{Ba}^{2+}$ from its weakly-attached xenon neighbors, so efficient capture of $\mathrm{Ba}^{2+}$ from high-pressure xenon gas is expected. Notably, the xenon solvation shell may also offer useful protection until proximity adequate for capture occurs.

A pertinent question for design of a molecular sensing layer at the gas-solid interface is the optimal surface density of probe molecules. An ultra-dense fluorophore layer is likely to suffer from collective quenching effects, whereas an overly sparse one may not efficiently capture arriving ions. To this end we undertook computations of the range of ion capture. Complexation can be considered to be inevitable when the ion-molecule proximity is such that the binding energy is more than a few times the thermal energy $(\mathrm{kT} \sim 0.02 \mathrm{eV}$ at STP $)$. To evaluate the effective capture range, the most stable geometry of the complex $\mathbf{1 B}-\mathbf{B a}^{2+}$ was evaluated as a function of ion-to-surface distance in gas phase (Fig. 8). Upon allowing $\mathrm{Ba}^{2+}$ to move closer to the azacrown ether surface, a bent geometry was obtained where the ion is bound to naphthalimide oxygen along with four ethereal oxygen of the azacrown ether substructure (structure 4). This bent geometry of the complex is consistent with the NMR titration study in solution, in which the nitrogen atom of the azacrown ether did not appear to directly bind with the electron-withdrawing $\mathrm{Ba}^{2+}$ ion.
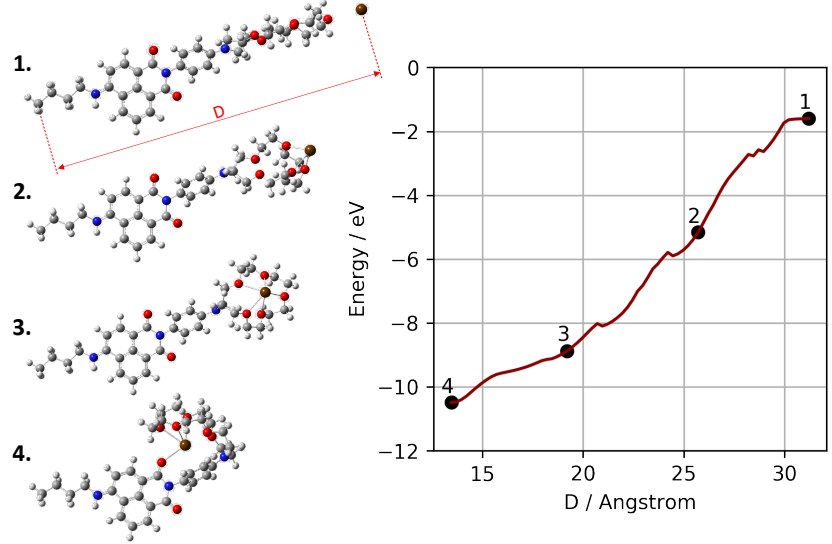

Figure 8: Simulation of ionic capture (left), and potential energy surface in the gas phase as a function of ion-to-surface distance (right)

Beyond a range of 32 Angstroms from the anchor point, our simulations fail to converge. The binding energy at this distance is still large, at $1.75 \mathrm{eV}$. Extrapolating the trend observed in the potential energy surface, it appears that the effective binding range of the molecule is somewhere between 32 and 40 Angstroms. This suggests a range of densities for future monolayer construction, in order to realize efficient 
binding and reduce intermolecular distortions to fluorescent events. Exploration of the power of sensitive, semi-dense monolayers for ion capture at the solid-gas interface is the next immediate step in this ongoing program.

\section{Conclusion}

We have designed, synthesized, and studied barium selective fluorescent sensors to image $\mathrm{Ba}^{2+}$ via SMFI in dry environments. We found that a new class of visible-spectrum 1,8-naphthalimide derivatives with diaza-18-crown-6 substituents dramatically improved the selectivity of the sensors to $\mathrm{Ba}^{2+}$ without significantly compromising sensitivity. Solution-phase fluorescence and NMR experiments supported a PET mechanism enabling turn-on fluorescence sensing in the presence of metal ions. Experiments showed strong binding of $\mathrm{Ba}^{2+}$ to the sensor with 1:1 stoichiometry and a nanomolar limit of detection. Experimentally validated theoretical calculations illuminated the mechanism of fluorescence sensing, in addition to providing insights into expected behaviour as gas-solid interfaces.

SMFI microscopy in air-coupled epifluorescent mode was employed for sensing of single $\mathrm{Ba}^{2+}$ ions with these fluorophores. Cyanoacrylate was found to be an effective support medium for the aforementioned fluorophores, and due to the sensor's strong selectivity for barium, no competitive binding agent was required to achieve single molecule sensitivity with these molecules. Single $\mathrm{Ba}^{2+}$ candidates were resolved spatially and identified through single-step photobleaching transitions, with enhanced prevalence in bariumwashed samples. Realization of single $\mathrm{Ba}^{2+}$ sensitivity under these conditions represents an important step toward practical application of this technique within time projection chambers. Such a technique could enable new precision and robustness in searches for $0 \nu \beta \beta$ in xenon gas.

\subsection{Acknowledgements}

This work was undertaken as part of an ongoing interdisciplinary program to develop barium tagging technologies for the NEXT experiment. We gratefully acknowledge support from the Department of Energy under awards DESC0019054 and DE-SC0019223, and from the University of Texas at Arlington. NMR experiments were made possible by support from the NSF, CHE-0840509 (CRIF: MU). Mass spectrometry experiments were completed in the Shimadzu Center for Analytical Chemistry at UT Arlington.

\section{References}

(1) Moe, M. K. New approach to the detection of neutrinoless double beta decay. Physical Review 1991, C44, 931-934.

(2) Chambers, C., et al. Imaging individual barium atoms in solid xenon for barium tagging in nEXO. Nature 2019, 569, 203-207.

(3) McDonald, A.; Jones, B.; Nygren, D.; Adams, C.; Álvarez, V.; Azevedo, C.; Benlloch-Rodríguez, J.; Borges, F.; Botas, A.; Cárcel, S., et al. Demonstration of single-barium-ion sensitivity for neutrinoless doublebeta decay using single-molecule fluorescence imaging. Physical review letters 2018, 120, 132504.

(4) Fukugita, M.; Yanagida, T. Baryogenesis Without Grand Unification. Phys. Lett. 1986, B174, 45-47.

(5) Nygren, D. R. Detection of the barium daughter in $136 \mathrm{Xe} \rightarrow 136 \mathrm{Ba}+2 \mathrm{e}-$ by in situ single-molecule fluorescence imaging. Nuclear Instruments and Methods in Physics Research Section A: Accelerators, Spectrometers, Detectors and Associated Equipment 2016, 824, $2-5$.

(6) Jones, B.; McDonald, A.; Nygren, D. Single molecule fluorescence imaging as a technique for barium tagging in neutrinoless double beta decay. Journal of Instrumentation 2016, 11, P12011.

(7) De Silva, A. P.; Gunaratne, H. N.; Gunnlaugsson, T.; Huxley, A. J.; McCoy, C. P.; Rademacher, J. T.; Rice, T. E. Signaling recognition events with fluorescent sensors and switches. Chemical reviews 1997, 97, $1515-1566$

(8) Valeur, B.; Leray, I. Design principles of fluorescent molecular sensors for cation recognition. Coordination Chemistry Reviews 2000, 205, 3-40.

(9) Hamilton, G. R.; Sahoo, S. K.; Kamila, S.; Singh, N.; Kaur, N.; Hyland, B. W.; Callan, J. F. Optical probes for the detection of protons, and alkali and alkaline earth metal cations. Chemical Society Reviews 2015, 44, 4415-4432.

(10) Duke, R. M.; Veale, E. B.; Pfeffer, F. M.; Kruger, P. E.; Gunnlaugsson, T. Colorimetric and fluorescent anion sensors: an overview of recent developments in the use of 1, 8-naphthalimide-based chemosensors. Chemical society reviews 2010, 39, 3936-3953.

(11) Panchenko, P. A.; Fedorova, O. A.; Fedorov, Y. V. Fluorescent and colorimetric chemosensors for cations based on 1, 8-naphthalimide derivatives: design principles and optical signalling mechanisms. Russian Chemical Reviews 2014, 83, 155.

(12) Panchenko, P. A.; Fedorov, Y. V.; Fedorova, O. A.; Jonusauskas, G. Comparative analysis of the PET and ICT sensor properties of 1, 8-naphthalimides containing aza-15-crown-5 ether moiety. Dyes and Pigments 2013, 98, 347-357. 
(13) Gao, Z.; Hao, Y.; Zheng, M.; Chen, Y. A fluorescent dye with large Stokes shift and high stability: synthesis and application to live cell imaging. Rsc Advances 2017, 7, 7604-7609.

(14) Alfimov, M.; Gromov, S. Applied Fluorescence in Chemistry, Biology and Medicine; Springer, 1999; pp 161-178.

(15) Hou, C.; Urbanec, A. M.; Cao, H. A rapid Hg2+ sensor based on aza-15-crown-5 ether functionalized 1 , 8-naphthalimide. Tetrahedron letters 2011, 52, 49034905.

(16) Panchenko, P.; Park, V.; Fedorova, O.; Fedorov, Y. V.; Kataev, E. Cation-dependent spectral properties of fluorescent complexon based on 1, 8-naphthalimide with PET mechanism of optical response. Russian Chemical Bulletin 2015, 64, 1871-1876.

(17) Panchenko, P. A.; Fedorov, Y. V.; Fedorova, O. A. Selective fluorometric sensing of $\mathrm{Hg} 2+$ in aqueous solution by the inhibition of PET from dithia-15crown-5 ether receptor conjugated to 4-amino-1, 8naphthalimide fluorophore. Journal of Photochemistry and Photobiology A: Chemistry 2018, 364, 124-129.

(18) Panchenko, P. A.; Polyakova, A. S.; Fedorov, Y. V.; Fedorova, O. A. Chemoselective detection of $\mathrm{Ag}+$ in purely aqueous solution using fluorescence 'turnon'probe based on crown-containing 4-methoxy-1, 8naphthalimide. Mendeleev Communications 2019, 29, $155-157$.

(19) Georgiev, N. I.; Dimitrova, M. D.; Todorova, Y. D.; Bojinov, V. B. Synthesis, chemosensing properties and logic behaviour of a novel ratiometric 1, 8naphthalimide probe based on ICT and PET. Dyes and Pigments 2016, 131, 9-17.

(20) Fernández-Alonso, S.; Corrales, T.; Pablos, J.; Catalina, F. Solid fluorescence sensors obtained by functionalization of photocrosslinked water-swollen acrylic membranes with 4-piperazine naphthalimide derivatives. Polymer 2017, 124, 139-150.

(21) Aderinto, S. O.; Zhang, H.; Wu, H.; Chen, C.; Zhang, J.; Peng, H.; Yang, Z.; Wang, F. Synthesis and studies of two proton-receptor fluorescent probes based on 1, 8-naphthalimide. Coloration Technology 2017, 133, 40-49.

(22) Georgiev, N. I.; Krasteva, P. V.; Bojinov, V. B. A ratiometric 4-amido-1, 8-naphthalimide fluorescent probe based on excimer-monomer emission for determination of $\mathrm{pH}$ and water content in organic solvents. Journal of Luminescence 2019, 212, 271-278.

(23) Licchelli, M.; Orbelli Biroli, A.; Poggi, A. A prototype for the chemosensing of $\mathrm{Ba} 2+$ based on self-assembling fluorescence enhancement. Organic letters 2006, 8, 915-918.

(24) Patnaik, P. Handbook of inorganic chemicals; McGrawHill New York, 2003; Vol. 529.
(25) Zhao, J.-M.; Zong, Q.-S.; Chen, C.-F. Complexation of triptycene-based macrotricyclic host toward (9-anthracylmethyl) benzylammonium salt: a $\mathrm{Ba} 2+$ selective fluorescence probe. The Journal of organic chemistry 2010, 75, 5092-5098.

(26) Saluja, P.; Kaur, N.; Singh, N.; Jang, D. O. A benzthiazole-based tripodal chemosensor for $\mathrm{Ba} 2+$ recognition under biological conditions. Tetrahedron letters 2011, 52, 6705-6708.

(27) Banerjee, T.; Suresh, M.; Ghosh, H. N.; Das, A. Competitive binding of $\mathrm{Ba} 2+$ and $\mathrm{Sr} 2+$ to 18 -Crown-6 in a Receptor with a 1-Methoxyanthraquinone Analogue as the Other Binding Site. European Journal of Inorganic Chemistry 2011, 2011, 4680-4690.

(28) Basa, P. N.; Bhowmick, A.; Schulz, M. M.; Sykes, A. G. Site-selective imination of an anthracenone sensor: selective fluorescence detection of barium (II). The Journal of organic chemistry 2011, 76, 7866-7871.

(29) Guo, H.; Kuwabara, T. Colorimetric Chemosensor for Barium Metal Ions Using Tris (bipyridinium-crown ether) Conjugate. Chemistry Letters 2013, 42, 194196.

(30) García Grajeda, B. A.; Soto Acosta, S. G.; Aguila, S. A.; Peinado Guevara, H.; Díaz García, M. E.; Cruz Enríquez, A.; Campos Gaxiola, J. J. Selective and colorimetric detection of $\mathrm{Ba} 2+$ ions in aqueous solutions using 11mercaptoundecylphosphonic acid functionalized gold nanoparticles. $R S C$ advances 2017,

(31) Chaichana, K.; Phutlaprungrueang, N.; Chaicharoenwimolkul, L.; Promkatkaew, M.; Kongsriprapan, S. A selective fluorescence probe based on naphthalene for the detection of barium (ii). Spectrochimica Acta Part A: Molecular and Biomolecular Spectroscopy 2019, 207, 118-122.

(32) Li, F.; Zhong, K.; Hou, S.; Tang, L.; Bian, Y. A simple and efficient fluorescent probe for detecting $\mathrm{Ba} 2+$ and its various applications. Tetrahedron Letters 2020, 61, 151558.

(33) Kravchenko, J.; Darrah, T. H.; Miller, R. K.; Lyerly, H. K.; Vengosh, A. A review of the health impacts of barium from natural and anthropogenic exposure. Environmental geochemistry and health 2014, 36, 797-814.

(34) Łukasik-Głębocka, M.; Sommerfeld, K.; Hanć, A.; Grzegorowski, A.; Barałkiewicz, D.; Gaca, M.; Zielińska-Psuja, B. Barium determination in gastric contents, blood and urine by inductively coupled plasma mass spectrometry in the case of oral barium chloride poisoning. Journal of analytical toxicology 2014, 38, 380-382.

(35) Nakahara, Y.; Kida, T.; Nakatsuji, Y.; Akashi, M. A novel fluorescent indicator for $\mathrm{Ba} 2+$ in aqueous micellar solutions. Chemical Communications 2004, 224225 . 
(36) Nakahara, Y.; Kida, T.; Nakatsuji, Y.; Akashi, M. Fluorometric sensing of alkali metal and alkaline earth metal cations by novel photosensitive monoazacryptand derivatives in aqueous micellar solutions. Organic $\&$ biomolecular chemistry 2005, 3, 1787-1794.

(37) Kondo, S.-i.; Takahashi, T.; Takiguchi, Y.; Unno, M. Synthesis and photophysical properties of a 2, 2'-bianthracene-based receptor bearing two aza-15crown-5 ethers for naked-eye detection of barium ion. Tetrahedron letters 2011, 52, 453-457.

(38) Yang, C.; Liu, L.; Zeng, T.; Yang, D.; Yao, Z.; Zhao, Y.; Wu, H.-C. Highly sensitive simultaneous detection of lead (II) and barium (II) with G-quadruplex DNA in $\alpha$-hemolysin nanopore. Analytical chemistry 2013, 85, 7302-7307.

(39) Xu, L.; Chen, Y.; Zhang, R.; Gao, T.; Zhang, Y.; Shen, X.; Pei, R. A highly Sensitive Turn-on Fluorescent Sensor for $\mathrm{Ba} 2+$ Based on G-Quadruplexes. Journal of fluorescence 2017, 27, 569-574.

(40) Ravichandiran, P.; Subramaniyan, S. A.; Bella, A. P.; Johnson, P. M.; Kim, A. R.; Shim, K. S.; Yoo, D. J. Simple fluorescence turn-on chemosensor for selective detection of $\mathrm{Ba} 2+$ ion and its live cell imaging. Analytical chemistry 2019, 91, 10095-10101.

(41) Monrabal, F.; Gómez-Cadenas, J.; Toledo, J.; Laing, A.; Álvarez, V.; Benlloch-Rodríguez, J.; Cárcel, S.; Carrión, J.; Esteve, R.; Felkai, R., et al. The next white (new) detector. Journal of Instrumentation 2018, 13, P12010.

(42) Martín-Albo, J.; Vidal, J. M.; Ferrario, P.; NebotGuinot, M.; Gómez-Cadenas, J.; Álvarez, V.; Azevedo, C.; Borges, F.; Cárcel, S.; Carrión, J., et al. Sensitivity of NEXT-100 to neutrinoless double beta decay. Journal of High Energy Physics 2016, 2016, 159 .

(43) Adams, C.; Alvarez, V.; Arazi, L.; Arnquist, I.; Azevedo, C.; Bailey, K.; Ballester, F.; BenllochRodriguez, J.; Borges, F.; Byrnes, N., et al. Sensitivity of a tonne-scale NEXT detector for neutrinoless double beta decay searches. arXiv preprint arXiv:2005.06467 2020,

(44) Thapa, P.; Arnquist, I.; Byrnes, N.; Denisenko, A.; Foss, F.; Jones, B.; McDonald, A.; Nygren, D.; Woodruff, K. Barium chemosensors with Dry-phase fluorescence for neutrinoless Double Beta Decay. Scientific reports 2019, 9, 1-13.

(45) Rivilla, I., et al. Towards a background-free neutrinoless double beta decay experiment based on a fluorescent bicolor sensor. arXiv:1909.02782 2019,

(46) Luk'yanenko, N.; Basok, S.; Kulygina, E. Y.; Bogashchenko, T. Y.; Yakovenko, I. Synthesis of monoazacrown ethers under phase-transfer catalysis. Russian Journal of Organic Chemistry 2012, 48, 1345-1352.
(47) Koonrugsa, N.; Fuangswasdi, S. Metal ion chemosensors based on diaza-18-crown-6 coupling with azobenzene dye. Spectrochimica Acta Part A: Molecular and Biomolecular Spectroscopy 2019, 215, 15-23.

(48) Zhao, Y.; Truhlar, D. G. The M06 suite of density functionals for main group thermochemistry, thermochemical kinetics, noncovalent interactions, excited states, and transition elements: two new functionals and systematic testing of four M06-class functionals and 12 other functionals. Theoretical Chemistry Accounts 2008, 120, 215-241.

(49) Dolg, M.; Wedig, U.; Stoll, H.; Preuss, H. Energyadjusted abinitio pseudopotentials for the first row transition elements. The Journal of chemical physics 1987, 86, 866-872.

(50) Kaupp, M.; Schleyer, P. v. R.; Stoll, H.; Preuss, H. Pseudopotential approaches to $\mathrm{Ca}, \mathrm{Sr}$, and $\mathrm{Ba}$ hydrides. Why are some alkaline earth MX2 compounds bent? The Journal of chemical physics 1991, 94, 1360-1366.

(51) Bergner, A.; Dolg, M.; Küchle, W.; Stoll, H.; Preuß, H. $\mathrm{Ab}$ initio energy-adjusted pseudopotentials for elements of groups 13-17. Molecular Physics 1993, 80, 1431-1441.

(52) Dolg, M.; Stoll, H.; Preuss, H.; Pitzer, R. M. Relativistic and correlation effects for element 105 (hahnium, $\mathrm{Ha})$ : a comparative study of $\mathrm{M}$ and $\mathrm{MO}(\mathrm{M}=\mathrm{Nb}, \mathrm{Ta}$, Ha) using energy-adjusted ab initio pseudopotentials. The Journal of Physical Chemistry 1993, 97, 58525859 .

(53) Weigend, F.; Ahlrichs, R. Balanced basis sets of split valence, triple zeta valence and quadruple zeta valence quality for $\mathrm{H}$ to $\mathrm{Rn}$ : Design and assessment of accuracy. Physical Chemistry Chemical Physics 2005, 7, 3297-3305.

(54) Weigend, F. Accurate Coulomb-fitting basis sets for $\mathrm{H}$ to Rn. Physical chemistry chemical physics 2006, 8, 1057-1065.

(55) Runge, E.; Gross, E. K. Density-functional theory for time-dependent systems. Physical Review Letters 1984, 52, 997.

(56) Marenich, A. V.; Cramer, C. J.; Truhlar, D. G. Universal solvation model based on solute electron density and on a continuum model of the solvent defined by the bulk dielectric constant and atomic surface tensions. The Journal of Physical Chemistry B 2009, 113, 6378-6396.

(57) Hall, R. J.; Davidson, M. M.; Burton, N. A.; Hillier, I. H. Combined density functional, selfconsistent reaction field model of solvation. The Journal of Physical Chemistry 1995, 99, 921-924.

(58) Gaussian09, R. A. 1, mj frisch, gw trucks, hb schlegel, ge scuseria, ma robb, jr cheeseman, g. Scalmani, v. Barone, b. Mennucci, ga petersson et al., gaussian. Inc., Wallingford CT 2009, 121, 150-166. 
(59) Wang, L.; Qin, W.; Tang, X.; Dou, W.; Liu, W.; Teng, Q.; Yao, X. A selective, cell-permeable fluorescent probe for $\mathrm{Al} 3+$ in living cells. Organic $\& 3$ biomolecular chemistry 2010, 8, 3751-3757.

(60) Lee, H.; Lee, H.-S.; Reibenspies, J. H.; Hancock, R. D. Mechanism of "turn-on" fluorescent sensors for mercury (II) in solution and its implications for ligand design. Inorganic chemistry 2012, 51, 10904-10915.

(61) Bainglass, E.; Jones, B. P.; Foss, F.; Huda, M.; Nygren, D. Mobility and Clustering of Barium Ions and Dications in High Pressure Xenon Gas. Phys. Rev. A 2018, 97, 062509. 